\title{
Ad Hoc Routing with Early Unidirectionality Detection and Avoidance ${ }^{\star}$
}

\author{
Young-Bae Ko ${ }^{1}$, Sung-Ju Lee ${ }^{2}$, and Jun-Beom Lee ${ }^{1}$ \\ 1 College of Information \& Communication, Ajou University, Suwon, Korea \\ 2 Mobile \& Media Systems Lab, Hewlett-Packard Laboratories, Palo Alto, CA 94304
}

\begin{abstract}
This paper is motivated by the observation that current research in ad hoc networks mostly assumes a physically flat network architecture with the nodes having homogeneous characteristics, roles, and networking- and processingcapabilities (e.g., network resources, computing power, and transmission power). In real-world ad hoc networks however, node heterogeneity is inherent. New mechanisms at the network layer are required for effective and efficient utilization of such heterogeneous networks. We discuss the issues and challenges for routing protocol design in heterogeneous ad hoc networks, and focus on the problem of quickly detecting and avoiding unidirectional links. We propose a routing framework called Early Unidirectionality Detection and Avoidance (EUDA) that utilizes geographical distance and path loss between the nodes for fast detection of asymmetric and unidirectional routes. We evaluate our scheme through ns- 2 simulation and compare it with existing approaches. Our results demonstrate that our techniques work well in these realistic, heterogeneous ad hoc networking environments with unidirectional links.
\end{abstract}

\section{Introduction}

Starting from the days of the packet radio networks (PRNET) [10,13] in the 1970s and survivable adaptive networks (SURAN) [11] in the 1980s to the global mobile (GloMo) networks [14] in the 1990s and the current mobile ad hoc networks (MANET) [6], the multi-hop ad hoc network has received great amount of research attention. The ease of deployment without any existing infrastructure makes ad hoc networks an attractive choice for applications such as military operations, disaster recovery, search-and-rescue, and so forth. With the advance of IEEE 802.11 technology and the wide availability of mobile wireless devices, civilians can also form an instantaneous ad hoc network in conferences or in class rooms.

Recent research in ad hoc networks has focused on medium access control and routing protocols. Because of shared wireless broadcast medium, contention and hidden terminals are common in ad hoc networks and hence MAC is an important problem. Routing is also an interesting issue as routes are typically multi-hop. When the end-toend source and destination are not within each other's transmission range, routes are multi-hop and they rely on intermediate nodes to forward the packets. The construction and maintenance of the routes are especially challenging when nodes are mobile.

\footnotetext{
* This work was in part supported by grant No. R05-2003-000-10607-02004 from Korea Science \& Engineering Foundation, and University IT Research Center project.
} 
Although there has been a great amount of work in these areas, most of the research assumes the nodes are homogeneous. All nodes are assumed to have the same or similar radio propagation range, processing capability, battery power, storage, and so forth. Even the schemes that utilize the hierarchy of the nodes [15, 25] assume a flat physical network structure and the hierarchies are merely logical. In reality however, nodes in ad hoc networks have heterogeneity. In the military scenarios for instance, the troop leader is usually equipped with more powerful networking devices than the private soldiers of the troop. Radios installed in the vehicles such as tanks and jeeps have more capabilities than radios the soldiers carry, as vehicles do not have the same size- or power-constraints as the mobile soldiers have. Another reason could be the financial cost. The state-ofthe-art equipments are very expensive and hence only a small number of nodes could be supplied with such high-end devices. Similarly, civilians possess different types of mobile devices ranging from small palm-pilots and PDAs to laptops.

The heterogeneity of the ad hoc network nodes creates challenges to current MAC and routing protocols. Many MAC protocols use the request-to-send/clear-to-send (RTS/CTS) handshake to resolve channel contention for unicast packets. The assumption here is that when node $A$ can deliver RTS to node $B$, node $A$ will also be able to receive CTS from node $B$. Routing protocols in ad hoc networks typically assume bidirectional, symmetric routes, which do not always hold true when node heterogeneity is introduced. The performance of these protocols may degrade in networks with heterogeneous nodes [23].

One of the major challenges in ad hoc networks with heterogeneous nodes is the existence of "unidirectional links." Along with the medium access control, routing performance can be suffered from the existence of unidirectional links and routes. Unidirectional links may exist for various reasons. Different radios may have different propagation range, and hence unidirectional links may exist between two nodes with different type of equipments. IEEE 802.11 b uses different transmission rates for broadcast and unicast packets. That creates gray zones [17] where nodes within that zone receive broadcast packets from a certain source but not unicast packets. The hidden terminal problem [28] can also result in unidirectional links. Moreover, interference, fading, and other wireless channel problems can affect the communication reachability of the nodes. Some recent proposals have nodes adjust the radio transmission range for the purpose of energy-aware routing [9] and topology control [26]. The nodes in these schemes transmit packets with the radio power just strong enough to reach their neighbors. When nodes move out of that range, the link turns into unidirectional, when in fact it could be bidirectional when each node sends packets with the maximum transmission range. The unidirectional links (and routes) are therefore, quite common in ad hoc networks.

In this paper, we focus on the issues and challenges for routing protocol design in heterogeneous ad hoc networks. Specifically, we focus on the heterogeneity of node transmission power and unidirectional links resulting from it. We propose a routing technique EUDA (Early Unidirectionality Detection and Avoidance) that proactively detects unidirectional links and avoids constructing routes that include such links. We introduce two approaches: (i) a network-layer solution that utilizes node location information and (ii) a cross-layer solution based on a path-loss model. 
The rest of the paper is organized as follows. Related work on heterogeneous ad hoc networks is covered in Section 2 . We then study how existing ad hoc routing protocols handle unidirectional links in Section 3. Section 4 introduces EUDA, followed by ns-2 simulation results in Section 5 . We conclude in Section 6 .

\section{Related Work}

There has been recent research interest in heterogeneous ad hoc networks. DEAR (Device and Energy Aware Routing) protocol [1] considers the heterogeneity of the nodes in terms of the power source. Nodes that have continuous energy supply from external power forward more packets than nodes that are running on battery. As the main goal of DEAR is energy-awareness and it only addresses power source heterogeneity, it does not investigate other issues such as unidirectional links that may result from the node heterogeneity. ISAIAH (Infra-Structure AODV for Infrastructured Ad Hoc networks) [16] introduces "pseudo base stations (PBS)" that are immobile and have infinite amount of power supply. Its routing protocol selects paths that include such PBS nodes instead of regular mobile nodes. Similar to DEAR, it does not address the problem of unidirectional routes. The notion of reliable nodes that are secure and robust to failure is used in [31]. This work focuses on the optimal placement of such reliable nodes and subsequent route construction. Heterogeneity of ad hoc network node is also studied in [30]. Initially, the nodes are grouped into clusters. Each cluster elects a backbone node based on node capabilities. The backbone nodes themselves form a network called Mobile Backbone Network (MBN) for efficient, scalable communication. The spirit is similar to existing hierarchical, clustering work, but it uses the "physical" hierarchy of the nodes. The optimal number of backbone nodes is obtained analytically, and the clustering and routing schemes are introduced. By using simulations, MAC performance in ad hoc networks with heterogeneous node transmission power is analyzed in [23]. This study illustrates the negative impact of unidirectional links on handshake-driven MAC protocols. Ad hoc network heterogeneity is also investigated in [3], but it only considers heterogeneous network interfaces.

There has been recent attention on routing in ad hoc networks with unidirectional links [2, 24, 27]. These schemes however, rely on proactive routing mechanisms where each node periodically exchanges link information for route maintenance. Various performance studies [4,7, 12] report that proactive table-driven routing protocols do not perform well in ad hoc networks, especially in highly mobile, dynamic situations. Although there have been numerous on-demand ad hoc routing protocols proposed, very few give attention to unidirectional links. Dynamic Source Routing (DSR) [8] could operate with unidirectional links, but it comes at the cost of excessive messaging overhead, as two network-wide flooding is required for each route construction; one from the source to the destination and the other from the destination to the source. Ad hoc On-Demand Distance Vector (AODV) [22] does not work well in the presence of unidirectional links. There have been proposals to solve this problem, which is the topic of the next section. 


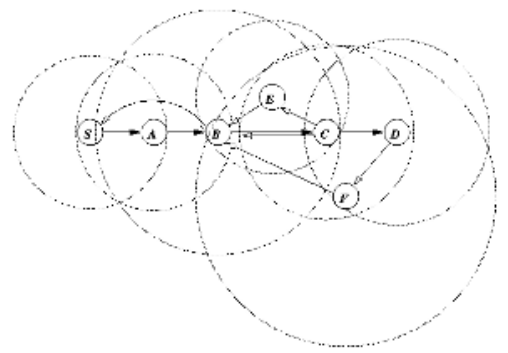

Fig. 1. Routing in networks with unidirectional links. The link between $\mathrm{B}$ and $\mathrm{C}$ is a unidirectional link. The packets transmitted by node B reach node $\mathrm{C}$, but not vice versa.

\section{Unidirectional Links in AODV}

On-demand routing is generally shown to give good performance than proactive schemes when node mobility is high and network connectivity changes frequently. Although our idea to be presented in Section 4 can be applied to proactive routing protocols, we focus here on on-demand routing. Here we illustrate how unidirectional routes are formed in existing on-demand routing approaches.

Nodes in on-demand routing protocols do not maintain routes to all nodes in the network. They build routes to only nodes they need to communicated with, only when they have data packets to send to. Routes are usually constructed by flooding a RouTE REQUEST (RREQ) packet to the entire network. When the destination receives multiple ROUTE REQUEST packets, it selects the best route based on the route selection algorithm (e.g., minimum delay, shortest hop, minimum energy, etc.). The destination then sends a Route REPLY (RREP) message to the source via the reverse of the chosen path. This ROUTE REPLY will reach the source of the route through the selected path only if the route is bidirectional. In addition to Route REPLY packets, Route ERROR (RERR) messages, which are used to inform the source node of the route disconnection, are transmitted through the reverse route. When there is a unidirectional link in the route, these on-demand routing protocols cannot operate correctly.

Let us investigate how existing, popular ad hoc on-demand routing protocols function in the presence of unidirectional links. AODV (Ad hoc On-Demand Distance Vector) routing algorithm [22] for example assumes that all links between neighboring nodes are symmetric (i.e., bi-directional links). Therefore, if there are unidirectional links in the network and these unidirectional links are included on a reverse path, AODV may not be successful in a route search. As an example in Figure 1 a RREQ packet generated by a source node $S$ traverses the path $\langle S-A-B-C-D>$ until it arrives at a destination node $D$. Each circle in the figure represents the node transmission range. When node $D$ receives the RREQ, it sends a RREP back to node $S$ via the reverse path, $<D-C-B-A-S>$. Note that however, the RREP is not able to reach from node $C$ to node $B$ because node $B$ is not located within node $C$ 's transmission range. As a result of a RREP delivery failure by node $C$, the source $S$ cannot receive the corresponding RREP packet and hence it experiences a route discovery failure in its first trial. Such a failure will repeatedly cause route discovery processes with no benefit. Although there 
exists a route that does not include any unidirectional link, $\langle S-A-B-E-C-D\rangle$, this route cannot be found as the shortest hop is one of the main route selection criteria in AODV. We refer to this scheme as the "Basic-AODV."

In the latest AODV specification [21, 18], some mechanisms are newly added to handle the problem of unidirectional links. One way to detect unidirectional links is to have each node periodically exchange hello messages that include neighboring information. This scheme however, requires large messaging overhead. Another solution is blacklisting. Whenever a node detects a unidirectional link to its neighbor, it blacklists that neighbor from which a link is unidirectional. Later when the node receives a RREQ from one of the nodes in its blacklist set, it discards the RREQ to avoid forming a reverse path with a unidirectional link. Each node maintains a blacklist and the entries in the blacklist are not source-specific. In order to detect a unidirectional link, a node sets the "Acknowledgment Required" bit in a RREP when it transmits the RREP to its next hop. On receiving this RREP with the set flag, the next hop neighbor returns an acknowledgment (also known as RREP-ACK) to the sending node to inform that the RREP was received successfully. In the case when RREP-ACK is not returned, the node puts its next hop node on its blacklist so that future RREQ packets received from those suspected nodes are discarded. We refer to this version of AODV as the "AODV-BL (Blacklist)" scheme.

Again, let's use Figure 1 to illustrate the AODV-BL scheme. Here, node $C$ cannot be acknowledged by node $B$ when delivering RREP and therefore it will put node $B$ in its blacklist set. Later when node $S$ re-broadcasts a new RREQ packet, node $C$ will ignore this RREQ received from $B$ but forward another copy of the RREQ from node $E$. Finally, a destination node $D$ will receive the RREQ through a longer route at this time. Node $D$ returns a RREP back to the source $S$ via a reverse path; in this case, the reverse path is $\langle D-C-E-B-A-S\rangle$, having no unidirectional links.

The AODV-BL scheme may be efficient when there are few unidirectional links. However, as the number of asymmetric links increase, its routing overhead is likely to become larger since a source node will always suffer from a failure in its first trial of route discovery and need to flood RREQ messages more than once to find a route with all bidirectional links. It also results in an increase of route acquisition delay.

\section{Ad Hoc Routing with EUDA}

\subsection{Basic Mechanism}

In EUDA (Early Unidirectionality Detection and Avoidance), a node detects a unidirectional link immediately when it receives a RREQ packet. Remember that in AODV-BL, such a detection will be done much later with its RREP-ACK and blacklisting mechanisms. Our goal is to detect a unidirectional link immediately in RREQ forwarding process. The basic idea is that, when node $X$ receives a RREQ from node $Y$, node $X$ compares its transmission range using the highest power level to an estimated distance between them. If the value of estimated distance from node $X$ to $Y$ is larger than the transmission range of node $X$, node $X$ considers its link to $Y$ as a unidirectional link, resulting in RREQ packet drop without any further forwarding. Only when a transmission range of node $X$ is equal to or larger than its estimated distance towards node $Y$, 
RREQs from $Y$ will be processed. In EUDA, all nodes receiving RREQ packet are required to decide whether to forward it or not. This decision is based on the comparison of transmission range with distance between the nodes. Note that duplicate detection is still enforced. If a node has already forwarded a RREQ from a source with a specific sequence number, it will drop the rest of the RREQs with the same $<$ source, sequence number $>$ entry. When a node has received RREQs but has not forwarded any of them (i.e., they are from nodes connected through a unidirectional link), it will process subsequent RREQs. Consequently, a node will forward at most one RREQ from a source with a specific sequence number.

Again, see Figure 1 as an example. When node $C$ receives a RREQ from node $B$, it detects a unidirectional link immediately using the proposed scheme and discards the packet. Later, node $C$ may receive another RREQ from node $E$. Node $C$ this time will forward this RREQ as it came through a bi-directional link. Finally, the destination $D$ receives the first RREQ via nodes $E$ and $C$, forming the reverse path $<D-C-E-$ $B-A-S>$ and replying its RREP through this path. Eventually, node $S$ obtains a path having only the bidirectional links. Note that in EUDA, this successful route discovery is achieved from the first attempt by the source, as long as there is at least one bidirectional route from a source to a destination. When no such route exists, the source will time-out without receiving any RREP. It will re-try few times before giving up when no routes can be found.

\subsection{Distance Estimation}

The next question is how to calculate an estimated distance between two communicating nodes, so that it can be compared with the radio transmission range to determine whether the link between two nodes are bidirectional or unidirectional. This can be done in two different ways as described below.

Network Layer Solution with Location Information. Suppose that the nodes know their geographical position 1 A transmitter node $X$ includes its own location information in RREQ to be broadcasted. When a node receives a RREQ from $X$, it calculates the estimated distance $(d)$ based on its own physical location. This method can be useful and is easy to implement, but requires information of physical location of the participating nodes.

Cross Layer Solution. As an alternative, we can utilize a wireless channel propagation model, i.e., the two-ray ground path loss model that is designed to predict the mean signal strength for an arbitrary transmitter-receiver separation distance. In wireless networks, if we know the transmitted signal power $\left(P_{t}\right)$ at the transmitter and a separation distance (d) of the receiver, the received power $\left(P_{r}\right)$ of each packet is given by the following equation:

\footnotetext{
${ }^{1}$ Commonly, each node determines its own location by using GPS or some other techniques for positioning service [5]. Recent research has shown that location information can be utilized for ad hoc routing protocols to improve their performance [19, 29].
} 
Table 1. Variables and their default values in ns-2.

\begin{tabular}{lll}
\hline Parameters & Definition & Default Value \\
\hline$G_{t}$ & Transmitter antenna gain & 1.0 \\
$G_{r}$ & Receiver antenna gain & 1.0 \\
$h_{t}$ & Transmitter antenna height & $1.5 \mathrm{~m}$ \\
$h_{r}$ & Receiver antenna height & $1.5 \mathrm{~m}$ \\
$L$ & System loss factor & 1.0 (i.e., no loss) \\
\hline
\end{tabular}

$$
P_{r}=\frac{P_{t} * G_{t} * G_{r} *\left(h_{t}^{2} * h_{r}^{2}\right)}{d^{4} * L}
$$

Table 1 lists all the variables used in the equation above and their default values commonly set in $n s-2$ simulations. Now we can derive the following equation from Eq. (1) to compute the distance:

$$
d=\sqrt[4]{\frac{P_{t} * G_{t} * G_{r} *\left(h_{t}^{2} * h_{r}^{2}\right)}{P_{r} * L}}
$$

The above Eq. (2) states that the distance between two communicating nodes can be estimated at a receiver side, if the transmitted power level $P_{t}$ of the packet transmitter and the power received at the receiver $P_{r}$ are known.

To implement this method, the transmitter should make the transmitted power information available to the receiver, by putting the power information either on a RREQ or a MAC frame. However, the latter approach may cause a compatibility problem with IEEE 802.11, as it has to modify the format of the MAC header in order to specify $P_{t}$. Therefore, we use the former approach of modifying a RREQ packet format.

\subsection{Discussion on the Distance Estimation Scheme}

One assumption behind the unidirectionality detection methods presented above is that any node's communication range is constant when it transmits with its maximum power $P_{\text {max }}$. Remind that this theoretically maximum transmission range is compared with the estimated distance between the two nodes to detect unidirectional links. In reality however, there may be some attenuation of the transmitter power over distance. Therefore, one would argue that such an assumption is unrealistic because transmission range of the RREQ receiver (thus, a potential RREP forwarder) can vary due to several negative environmental factors such as obstacles, reflections, fading, etc.

To take this argument into account, we modify the proposed distance estimation based comparison method so that unidirectional link detection is made with more realistic parameters of the channel gain, the receiver sensitivity, and the receiver's signal-to-noise ratio.

Let us assume that there are two nodes $i$ and $j$. When node $j$ receives a RREQ from node $i$, it measures the received signal power, $P_{r}(j)$. The channel gain, $G_{i j}$, is computed as the received power $\left(P_{r}(j)\right)$ at node $j$ over the transmitted power $\left(P_{t}(i)\right)$ at node $i$ (see Eq. (3) below): 


$$
G_{i j}=\frac{P_{r}(j)}{P_{t}(i)}
$$

We assume that the sender power $P_{t}(i)$ is advertised in the RREQ packet. We also assume that the channel gain $G_{i j}$ between two nodes $i$ and $j$ is approximately the same in both directions - note that the same assumption was also made in [20]. Given the transmitter/receiver power information and the channel propagation characteristics, the received power at node $i$ must at least be equal to its minimum receiving threshold $R X_{-}$Thres $_{i}$, in order for node $i$ to receive any packet successfully from node $j$ with the transmission power $P_{t}(j)$.

$$
P_{r}(i)=G_{i j} * P_{t}(j) \geq R X_{-} \text {Thresh }_{i}
$$

This implies that if node $j$ transmits at the maximum power $P_{t}(\max )$ (i.e., replacing $P_{t}(j)$ ) satisfies Eq. (4), it can successfully deliver packets to node $i$. Observe that the value of $R X_{-}$Thresh $_{i}$ is related to the receiving sensitivity at node $i$.

We now define one additional equation such that the observed signal-to-noise ratio $S N R_{i}$ for the transmission at node $i$ must at least be equal to its minimum $S N R \_T h r e s h_{i}$ (representing the channel status observed at node $i$ ):

$$
S N R_{i}=\frac{G_{i j} * P_{t}(j)}{P_{n}(i)} \geq S N R_{-} \text {Thresh }_{i}
$$

where $P_{n}(i)$ is the total noise node $i$ observes on the channel. Again, this implies that node $j$ can successfully transmit to $i$ when $j$ with its maximum transmission power satisfies Eq. (5).

To summarize, if the above two equations (Eqs. (4) and (5)) are satisfied when one node receives a RREQ from another node, these two nodes are considered to be able to communicate directly with each other and hence have a bi-directional link. Otherwise, it can be concluded as having a unidirectional link between them. With this modification, we have our scheme work better in more realistic scenarios, with its improved estimation accuracy. Nevertheless, there is a clear tradeoff between accuracy and complexity in estimation. Furthermore, the RREQ packet size needs to be increased to include additional information. The transmitter node $i$ of a RREQ packet is now required to include more information (i.e., its transmitted power $P_{t}(i)$, observed total noise $P_{n}(i)$, minimum received power threshold $R X_{-}$Thres $h_{i}$, and minimum signalto-interference ratio $S N R \_$Thresh $h_{i}$ ) on the RREQ packet.

\section{Performance Evaluation}

Although the EUDA framework can be applied to any ad hoc routing protocol, for performance evaluation purposes, we add the EUDA framework to AODV to simulate AODV-EUDA and compare it with the Basic-AODV and AODV with Black Listing (AODV-BL) protocols. As explained in the previous section, Basic-AODV does not include any technique for handling unidirectional links, whereas AODV-BL reactively avoids unidirectional links by using a blacklist set. In AODV-BL, the next hop of a failed 
RREP packet is inserted by a node detecting the RREP delivery failure. We performed a simulation study using an extended version of the network simulator $n s-2 . n s-2$ is a discrete event-driven network simulator with extensive support for simulation of TCP, routing, and multicast protocols. The extensions implemented by CMU Monarch project were used for our simulations. Their extensions enable simulation of multi-hop wireless ad hoc networks. Extensions include simulation modules for the IEEE 802.11 MAC protocol and the two-ray radio propagation model. For AODV-EUDA, we simulated both approaches (i.e., the network layer approach and the cross-layer approach) and they gave the same performance as the ns- 2 simulator only provides an ideal environment where the node location and path loss information are always correct.

\subsection{Simulation Environment}

In our simulation model, initial node locations ( $\mathrm{X}$ and $\mathrm{Y}$ coordinates) are obtained using a uniform distribution. All 100 nodes in the network move around in a rectangular region of size $1500 m \times 300 m$ according to the following mobility model. Each node chooses a direction, moving speed, and distance of move based on a predefined distribution and computes its next position $P$ and the time instant $T$ of reaching that position. Once the node reaches this destined position, it stays there for pause time and repeats the process. We always use zero pause time (i.e., continuous mobility), and two maximum speeds: $1 \mathrm{~m} / \mathrm{s}$ and $20 \mathrm{~m} / \mathrm{s}$. Thus, each node is assumed to move in a continuous fashion, at a random speed chosen from the interval $[0 \mathrm{~m} / \mathrm{s},(1$ or 20$) \mathrm{m} / \mathrm{s}]$. Our total simulation time is 900 seconds and we repeated each scenario ten times with different random seed numbers. In our experiments, the transmission range of a node is defined as either one of the two different values (250 meters corresponding to long ranges and 125 meters corresponding to short ranges). We modified the $n s-2$ to implement these variable transmission ranges and model unidirectional links between nodes. The wireless link bandwidth is $2 \mathrm{Mb} / \mathrm{s}$. Traffic pattern we used consists of 10 CBR connections running on UDP. Each CBR source generates four 512-byte data packets every second.

\subsection{Simulation Results}

Figure 2 shows the number of unidirectional links in the entire network as a function of varying fraction of low power nodes. The most number of unidirectional links exist when the fraction is 0.5 (thus, 50 nodes with transmission range of $250 \mathrm{~m}$ and the other 50 nodes with range $125 \mathrm{~m}$ ). A similar observation was also made in [18]. We performed experiments with different maximum speed of mobile nodes at $1 \mathrm{~m} / \mathrm{s}$ and $20 \mathrm{~m} / \mathrm{s}$, but the results are nearly identical. This result shows that the number of unidirectional links are influenced more by the low power node fraction rather than mobility, and the fraction remains the same between scenarios with different maximum speeds at a pause time of zero.

Figure 3 presents the packet delivery ratio of the three schemes we simulate, as a function of fraction of low power nodes. Packet delivery ratio is defined as the ratio of the number of data packets received by the CBR sinks and the number of data packets originated by the application layer CBR sources. We report the average over $10 \mathrm{CBR}$ connections between source-destination pairs. Note that the $y$-axis scale in these figures 


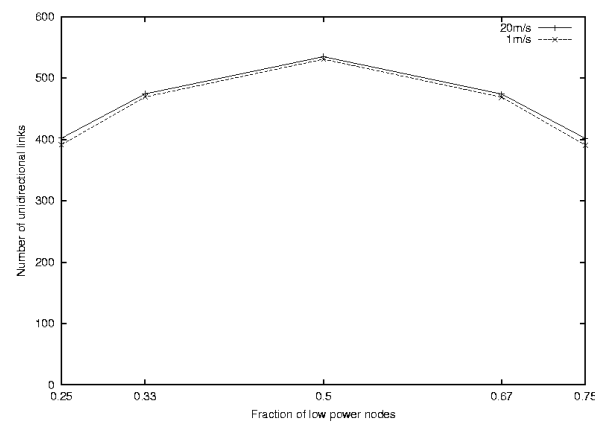

Fig. 2. Number of unidirectional links with varying fraction of low power nodes.

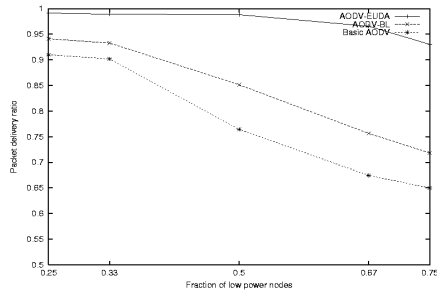

(a) Max speed: $1 \mathrm{~m} / \mathrm{s}$.

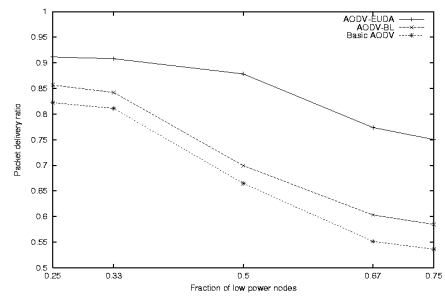

(b) Max speed: $20 \mathrm{~m} / \mathrm{s}$.

Fig. 3. Packet delivery ratio with varying fraction of low power nodes.

range from 0.5 to 1 . In both subfigures with different mobility speeds, as the fraction of low power nodes increases, the packet delivery ratio decreases for all protocols. Even the delivery ratio for the Basic-AODV, which does not handle unidirectional links, drops monotonically, even though there are the most unidirectional links when the fraction is 0.5 . This is somewhat unexpected, because with the increase in unidirectional links, Basic-AODV scheme is expected to experience more route discovery failures and show less packet delivery success rate. By analyzing the traces, we found that the network connectivity becomes poor with the increase in fraction of low power nodes as they have short transmission range. With a shorter transmission range, the likelihood of being connected with other nodes will decrease. Consequently, with the decrease in connectivity, the packet delivery ratio decreases as well because there are less number of routes available. Figure 4 shows the total number of neighbors per node as a function of low power node fraction. The total number of neighbors reflects the level of connectivity in the network - the larger the number of neighbors per node, the higher the number of paths between each nodes.

Back to Figure 3 with this observation in mind, we see that the drop in packet delivery ratio is much less drastic and the success delivery rate is consistently higher for AODVEUDA compared with the other two AODV schemes. This improvement of AODVEUDA is due to efficient and fast detection of unidirectional links. With AODV-EUDA, a route search failure will not occur even when there is unidirectional path from a source 


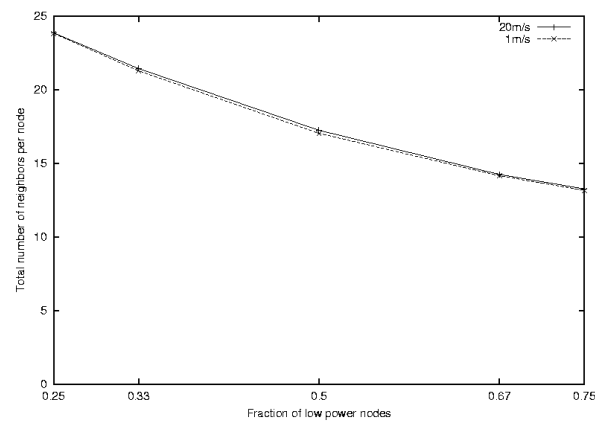

Fig. 4. Total Number of Neighbors per Node with varying fraction of low power nodes.

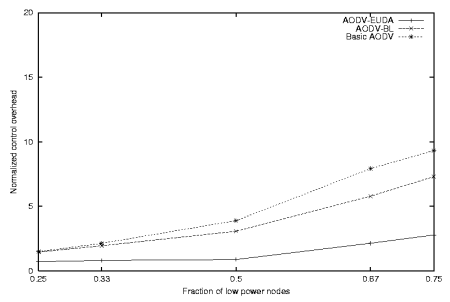

(a) Max speed: $1 \mathrm{~m} / \mathrm{s}$.

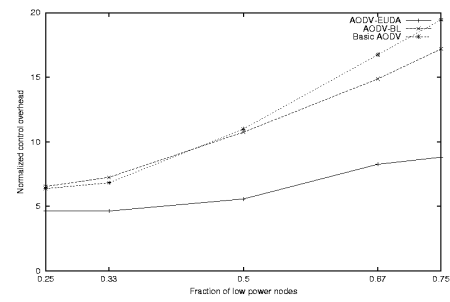

(b) Max speed: $20 \mathrm{~m} / \mathrm{s}$.

Fig. 5. Normalized control overhead with varying fraction of low power nodes.

to a destination. The Basic-AODV performs poorly in most cases as it does not take notice of unidirectional links and repeatedly performs route re-discoveries. As the Basic-AODV always chooses the shortest path between a source/destination pair, it cannot transmit any data packets when the shortest path includes one or more unidirectional links. The AODV-BL delivered less data compared with AODV-EUDA, but it still performed better than the Basic-AODV. Although AODV-BL detects unidirectional links, it only does so after a delivery failure and hence requires another route discovery process. AODVEUDA on the other hand, finds unidirectional links during the RREQ propagation phase and avoids including them in the route in the first route discovery attempt.

Mobility also affects the protocol performance. As expected, packet delivery fraction degrades for all protocols with increase in mobility as there are more route breaks (see Figure 3 (a) and (b), at the maximum speeds of $1 \mathrm{~m} / \mathrm{s}$ and $20 \mathrm{~m} / \mathrm{s}$, respectively). AODVEUDA continues to perform significantly better than the other two schemes.

Figure 5 shows the normalized routing overhead with varying fraction of low power nodes. We define the normalized routing overhead as the ratio between the total number of routing control packets transmitted by all nodes and the total number of data packets received by the destinations. Overall, AODV-EUDA has the lowest overhead compared with AODV-BL and the Basic-AODV. AODV-BL and the basic AODV perform excessive flooding as they can neither detect unidirectional links or detect them in a timely fashion. Such an excessive flooding clearly contributes to a larger routing overhead. 


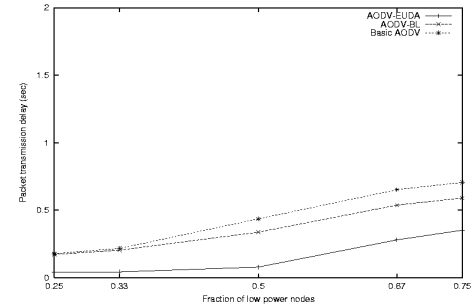

(a) Max speed: $1 \mathrm{~m} / \mathrm{s}$.

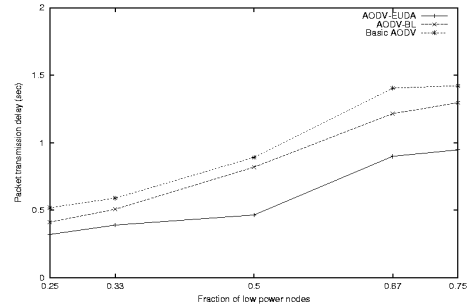

(b) Max speed: $20 \mathrm{~m} / \mathrm{s}$.

Fig. 6. End-to-end delay with varying fraction of low power nodes.

AODV-EUDA shows better efficiency because of its unique ability of early detection and avoidance of unidirectional links. As can be seen in Figure 5 (a) and (b), the routing overhead is also affected by mobility. The number of routing packet transmissions increases for all routing protocols with the increase in the speed of mobile nodes. With more dynamic mobility, route recoveries are more frequent as wireless links will break more often. We can still observe that AODV-EUDA provides a lower rate of increase than the other two schemes.

In Figure 6 we report the average end-to-end delay of successfully delivered data packets. The end-to-end delay is measured for the time from when a source generates a data packet to when a destination receives it. Therefore, this value includes all possible delay such as a buffering delay during route discovery, queuing and MAC delay during packet transmission, and propagation delay. The result again shows that AODVEUDA yields a significantly better performance (i.e., smaller end-to-end latency) than other protocols for both cases of two different maximum node speeds. This shows that AODV-EUDA effectively overcomes unidirectional links. By exploring the early unidirectionality detection and avoidance feature, AODV-EUDA is able to shorten the route discovery latency and hence the overall end-to-end delay. Note that route (re)discovery latency may dominate the total end-to-end delay. For this reason, the Basic-AODV and AODV-BL consistently showed poor delay performance. When any shortest path includes a unidirectional link, the sources running the Basic-AODV experience significant amount of route discovery delay as they cannot receive corresponding RREP packets until such unidirectional links disappear by some network topology change. AODV-BL also shows a poor delay performance compared with AODV-EUDA because it produces more number of RREQ packets to find bidirectional routes.

The effect of varying the moving speed of nodes is depicted more in detail in the following figures. The fraction of low power nodes is fixed to 0.5 as this value produces the largest number of unidirectional links. From Figures 7 to 9 we again see that AODVEUDA gives the best performance for all moving speeds in terms of packet delivery, routing overhead, and latency. With higher mobility, the frequency of route breaks increases and consequently packet delivery ratio becomes lower while routing overhead increases. AODV-EUDA has a lower increasing rate compared with the other schemes as its number of route requests is significantly reduced by preventing route discovery failures. With the Basic-AODV and AODV-BL, the existence of unidirectional links 


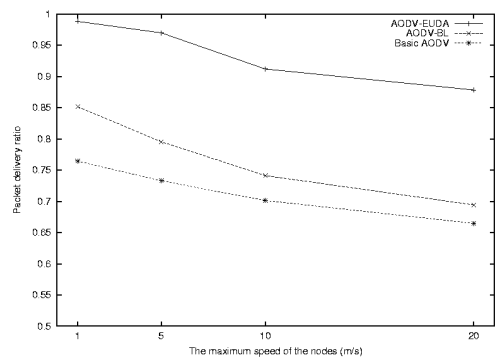

Fig. 7. Packet delivery ratio as a function of the maximum speed of the nodes.

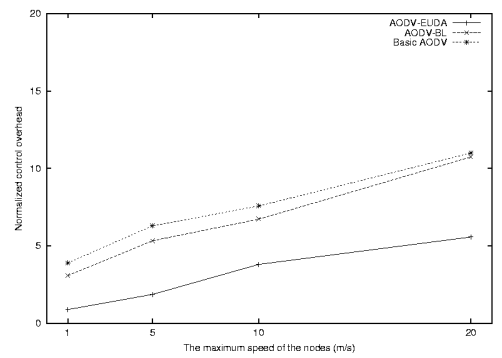

Fig. 8. Normalized control overhead as a function of the maximum speed of the nodes.

cause the failure of RREP delivery and hence the number of route requests is increased. It is interesting to see that AODV-BL has similar normalized routing overhead to that of the Basic-AODV (see Figure 8). This is due to the fact that AODV-BL transmits additional RREP-ACK packets to detect unidirectional links. Moreover, if the AODV-BL scheme fails to deliver RREP-ACK, it works exactly as the Basic-AODV. The delay of the AODV-EUDA is $100 \%$ to $300 \%$ smaller than that obtained by the Basic-AODV and AODV-BL schemes.

\section{Conclusion and Future Work}

We discussed node heterogeneity of real ad hoc network settings and studied a specific problem of unidirectional links that result from variance in radio transmission power. We addressed the limitations of existing routing protocols in the presence of unidirectional links. We proposed EUDA that detects unidirectional links in a timely fashion and excludes such links from being part of end-to-end communication paths. Two approaches have been considered. The first scheme uses node location information to estimate unior bi-directionality of a link while the second scheme utilizes a path loss model. Our simulation results have shown that when EUDA is applied to AODV, compared with AODV and AODV blacklisting, it gives superior throughput, less overhead with high efficiency, and shorter latency. Early detection of unidirectional links enables EUDA to use less messaging overhead and deliver more data packets with less delay. 


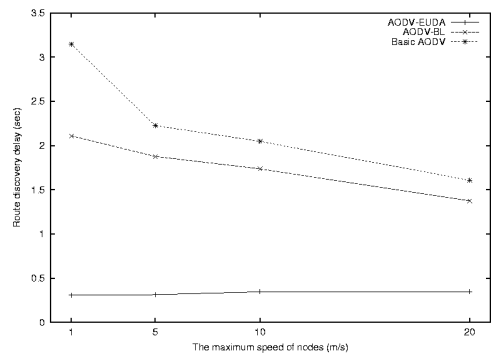

Fig. 9. End-to-end delay as a function of the maximum speed of the nodes.

Although EUDA is shown to work well, we could not truely evaluate our scheme as the ns-2 simulator provides rather optimistic channel propagation model. Our future work includes performing simulations with a more realistic path loss model and investigating further on the cross-layer approach where new MAC scheme is applied. Consideration of node heterogeneity other than radio transmission power, such as power source, antenna type, computing power are also ongoing. We are also building a network testbed with heterogeneous mobile devices for further performance study.

\section{References}

1. A. Avudainayagam, W. Lou, and Y. Fang, "DEAR: A device and energy aware routing protocol for heterogeneous ad hoc networks," Journal of Parallel and Distributed Computing, vol. 63, no. 2, pp. 228-236, Feb. 2003.

2. L. Bao and J. J. Garcia-Luna-Aceves, "Link state routing in networks with unidirectional links," in Proc. of IEEE ICCCN, Boston, MA, Oct. 1999.

3. J. Broch, D. A. Maltz, and D. B. Johnson, "Supporting hierarchy and heterogeneous interfaces in multi-hop wireless ad hoc networks," in Proc. of the IEEE Workshop on Mobile Computing, (I-SPAN), Perth, Western Australia, June 1999.

4. J. Broch, D. A. Maltz, D. B. Johnson, Y.-C. Hu, and J. Jetcheva, "A performance comparison of multi-hop wireless ad hoc network routing protocols," in Proc. of ACM MobiCom, Dallas, TX, Oct. 1998, pp. 85-97.

5. S. Capkun, M. Hamdi, and J.-P. Hubaux, "GPS-free positioning in mobile ad-hoc networks," in Proc. of HICSS, Hawaii, Jan. 2001, pp. 3481-3490.

6. IETF mobile ad hoc networks (MANET) working group charter. [Online]. Available: http://www.ietf.org/html.charters/manet-charter.html

7. P. Johansson, T. Larsson, N. Hedman, B. Mielczarek, and M. Degermark, "Scenario-based performance analysis of routing protocols for mobile ad-hoc networks," in Proc. of ACM MobiCom, Seattle, WA, Aug. 1999, pp. 195-206.

8. D. Johnson and D. Maltz, "Dynamic source routing in ad hoc wireless networks," in Mobile Computing, T. Imielinski and H. Korth, Eds. Kluwer Publishing Company, 1996, ch. 5, pp. 153-181.

9. C. E. Jones, K. M. Sivalingam, P. Agrawal, and J. C. Chen, "A survey of energy efficient network protocols for wireless networks," ACM/Kluwer Wireless Networks, vol. 7, no. 4, pp. 343-358, 2001.

10. R. E. Kahn, S. A. Gronemeyer, J. Burchfiel, and R. C. Kunzelman, "Advances in packet radio technology,” Proc. IEEE, vol. 66, no. 11, pp. 1468-1496, Nov. 1978. 
11. G. S. Lauer, "Hierarchical routing design for SURAN," in Proc. of IEEE ICC, Toronto, Canada, June 1986, pp. 93-102.

12. S.-J. Lee, M. Gerla, and C.-K. Toh, "A simulation study of table-driven and on-demand routing protocols for mobile ad hoc networks," IEEE Network, vol. 13, no. 4, pp. 48-54, July/Aug. 1999.

13. B. M. Leiner, D. L. Nielson, and F. A. Tobagi, "Issues in packet radio network design," Proc. IEEE, vol. 75, no. 1, pp. 6-20, Jan. 1987.

14. B. M. Leiner, R. J. Ruth, and A. R. Sastry, "Goals and challenges of the DARPA GloMo program,” IEEE Personal Commun. Mag., vol. 3, no. 6, pp. 34-43, Dec. 1996.

15. C. R. Lin and M. Gerla, "Adaptive clustering for mobile wireless networks," vol. 15, no. 7, pp. 1265-1275, Sept. 1997.

16. A. Lindgren and O. Schelen, "Infrastructured ad hoc networks," in Proc. of IWAAN, Vancouver, Canada, Aug. 2002, pp. 64-70.

17. H. Lundgren, E. Nordstrom, and C. Tschudin, "Coping with communication gray zones in ieee 802.11b based ad hoc networks," in Proc. of ACM WoWMoM, Atlanta, GA, Sept. 2002, pp. 49-55.

18. M. K. Marina and S. R. Das, "Routing performance in the presence of unidirectional links in multihop wireless networks)," in Proc. of ACM MobiHoc, Lausanne, Switzerland, June 2002, pp. 12-23.

19. M. Mauve, J. Widmer, and H. Hartenstein, "A survey on position-based routing in mobile ad hoc networks," IEEE Network, vol. 15, no. 6, pp. 30-39, Nov. 2001.

20. J. Monks, V. Bharghavan, and W.-M. Hwu, "A power controlled multiple access protocol for wireless packet networks," in Proc. of IEEE INFOCOM, Anchorage, AK, Mar. 2001.

21. C. Perkins, E. Belding-Royer, and S. Das, "Ad hoc on-demand distance vector (AODV) routing," IETF, RFC 3561, July 2003.

22. C. Perkins and E. Royer, "Ad-hoc on-demand distance vector routing," in Proc. of IEEE WMCSA, New Orleans, LA, Feb. 1999, pp. 90-100.

23. N. Poojary, S. V. Krishnamurthy, and S. Dao, "Medium access control in a network of ad hoc mobile nodes with heterogeneous power capabilities," in Proc. of IEEE ICC, Helsinki, Finland, June 2001.

24. R. Prakash, "A routing algorithm for wireless ad hoc networks with unidirectional links," ACM/Kluwer Wireless Networks, vol. 7, no. 6, pp. 617-625, Nov. 2001.

25. R. Ramanathan and M. Steenstrup, "Hierarchically-organized, multihop mobile wireless networks for quality-of-service support," ACM/Baltzer Mobile Networks and Applications, vol. 3 , no. 1, pp. 101-119, June 1998.

26. R. Ramanathan and R. Rosales-Hain, "Topology control of multihop wireless networks using transmit power adjustment," in Proc. of IEEE INFOCOM, Tel Aviv, Israel, Apr. 2000, pp. 404-413.

27. V. Ramasubramanian, R. Chandra, and D. Mosse, "Providing a bidirectional abstraction for unidirectional ad hoc networks," in Proc. of IEEE INFOCOM, New York, NY, June 2002.

28. F. A. Tobagi and L. Kleinrock, "Packet switching in radio channels: Part II-the hidden terminal problem in carrier sense multiple-access and the busy-tone solution," IEEE Trans. Commun., vol. COM-23, no. 12, pp. 1417-1433, Dec. 1975.

29. Y.-C. Tseng, S.-L. Wu, W.-H. Liao, and C.-M. Chao, "Location awareness in ad hoc wireless mobile networks," IEEE Computer, vol. 34, no. 6, pp. 46-52, June 2001.

30. K. Xu, X. Hong, and M. Gerla, "An ad hoc network with mobile backbones," in Proc. of IEEE ICC, New York, NY, Apr. 2002.

31. Z. Ye, S. V. Krishnamurthy, and S. K. Tripathi, "A framework for reliable routing in mobile ad hoc networks," in Proc. of IEEE INFOCOM, San Francisco, CA, Apr. 2003. 